\title{
Atoga No Mangareva, histoire mangarévienne. Regards croisés sur le Rongo de Cahors
}

Sarah Mohamed-Gaillard

\section{CpenEdition}

12 Journals

Édition électronique

URL : http://journals.openedition.org/jso/5985

DOI : $10.4000 /$ jso. 5985

ISSN : $1760-7256$

Éditeur

Société des océanistes

\section{Édition imprimée}

Date de publication : 15 décembre 2009

Pagination : $345-346$

ISBN : 978-2-85430-026-0

ISSN : 0300-953x

Référence électronique

Sarah Mohamed-Gaillard, « Atoga No Mangareva, histoire mangarévienne. Regards croisés sur le Rongo de Cahors », Journal de la Société des Océanistes [En ligne], 129 | juillet-décembre 2009, mis en ligne le 15 décembre 2009, consulté le 23 septembre 2020. URL : http://journals.openedition.org/jso/5985 ; DOI : https://doi.org/10.4000/jso.5985 
remarquablement bien ce peuple «austronésien » dont les voisins, au nord, au sud et à l'ouest, parlent des langues papoues. S'appuyant largement sur la littérature antérieure (dont les rapports des premiers missionnaires qu'il avait si attentivement étudiés et publiés dans ses publications précédentes), il souligne les principaux effets des changements culturels et matériels survenus dans cette vallée, non seulement depuis son premier séjour du printemps 1958, mais même depuis les premiers contacts avec les Blancs venus chercher de l'or puis l'installation d'une mission luthérienne en 1911. C'est néanmoins l'attention qu'il porta en 1965 à des tessons semi-enterrés dans un jardin wampar, comme il en avait trouvé en $1958 \mathrm{chez}$ les Watut, qui conduisit ses informateurs à les attribuer soit à des ancêtres reculés, soit à des êtres humains disparus mais aux esprits toujours redoutables, et l'amena finalement lui-même à cette recherche. Il y rassemble ses observations personnelles et celles de chercheurs plus récents, les données disponibles dans les collections des musées allemands, et avance (pp. 126-127) de très importantes hypothèses sur la diffusion de la poterie dans cette région, laissant entrevoir des échanges de nombreux autres éléments de culture, particulièrement avec les Anga et les Adzera. Les cinq observations de méthode concluant l'article sont évidemment à lire, venant d'un savant si distingué, et qui signe là une étude exemplaire de densité et d'intérêt.

\section{RÉFÉRENCES CITÉES}

FISCHER Hans, 1963. Watut. Notizen zur Kultur eines Melanesier-Stammes in Nordost-Neuguinea, Braunschweig, Albert Limbach Verlag.

-, 1968. Negwa. Eine Papua-Gruppe im Wandel, München, Klaus Renner Verlag.

- (Hg.), 1978. Wampar. Berichte über die alte Kultur eines Stammes in Papua New Guinea, Bremen, Im Selbstverlag des Übersee-Museums.

Gilles BounOuRE

Collectif, 2009. Atoga No Mangareva, histoire mangarévienne. Regards croisés sur le Rongo de Cahors, université Toulouse-Le Mirail, master Patrimoine 2008-2009, musée de Cahors Henri-Martin, 111 p., bibliographie, cartes, illus. couleur.

Ce bel ouvrage est né de la redécouverte, en 2000, dans les réserves du musée de Cahors Henri-Martin, d'une statuette faussement classée comme une divinité néo-calédonienne. La pièce, donnée au musée en 1835 par Joseph Bonafous-Murat, fut identifiée par Laurent Guillaut (conservateur en chef du musée de Cahors) et Claude Stefani (alors attaché de conservation du patrimoine au musée des Beaux-Arts de Chartres) comme une représentation anthropomorphique d'une divinité polynésienne, Rongo, divinité qui se trouve être liée à la pluie, à la fertilité et à l'abondance.
La découverte est importante. Haute de $73 \mathrm{~cm}$, cette statuette, taillée dans une pièce de bois de tamanu, est en effet la sixième représentation connue du dieu Rongo et la seule à être parée d'un pagne (voir la contribution d'Hélène Guiot, pp. 47-49). Le Rongo fut l'une des pièces maîtresses de l'exposition que le Musée du quai Branly consacra, en 2009, à « Mangareva, panthéon de Polynésie ».

Laurent Guillaut ouvrit alors l'enquête pour comprendre l'histoire de cette statue et son parcours de Mangareva, île de l'archipel des Gambier, à Cahors. Cette recherche fut couronnée par l'organisation d'une exposition au Musée Bonafous-Murat de Cahors et l'édition d'un ouvrage confiée à la promotion 2009 du master Patrimoine de Cahors, rattaché à l'université Toulouse-Le Mirail. À travers neuf communications richement illustrées, ce beau livre rend accessible au grand public l'histoire encore peu connue de cette île des Gambier et, au-delà, révèle l'histoire croisée de deux " pays » de l'Empire français. C'est donc un récit à plusieurs voix que propose cette histoire mangarévienne.

L'archipel des Gambier, sans doute peuplé depuis le $\mathrm{X}^{\mathrm{e}}$ siècle, fut aperçu et nommé par le capitaine du Duff, le 24 mai 1797. Mais l'équipage de ce navire, conduisant les premiers missionnaires de la London Missionary Society en Polynésie, ne toucha pas terre, découragé par l'attitude hostile des insulaires. La société mangarévienne était alors fortement hiérarchisée. Tara Hiquily, dont l'article (pp. 15-23) utilise largement les observations, quoique imparfaites, du père Honoré Laval (1968) et les travaux de Peter Buck (1938), souligne la complexité de l'organisation sociale, religieuse et matérielle de l'île. Bien que difficile d'accès et à l'écart des grandes routes de navigation traversant l'océan Pacifique, l'archipel des Gambier fut visité en 1825 par l'équipage du Blossom, navire anglais commandé par Frederick William Beechey. Ce premier contact, comme ce fut le cas dans de nombreuses autres îles d'Océanie, donna lieu à des tirs de la part des Européens pour punir les vols commis par les insulaires.

Après les navigateurs, arrivèrent en 1834 les missionnaires qui découvrirent une population déjà affectée par les maladies introduites dans l'archipel par les Européens. Les pères Caret et Laval, missionnaires de la congrégation des Sacrés-Cœurs de Picpus, baptisèrent dès 1836 l'akariki (le chef) Maputeoa, ce qui entraîna la conversion de l'ensemble de l'île. L'archipel des Gambier devint dès lors un bastion catholique en Polynésie. Parallèlement à l'action missionnaire et en réaction à l'annexion de la Nouvelle-Zélande par la Grande-Bretagne en 1840, la France plaça, cette même année, l'archipel sous son protectorat mais l'acte ne fut ratifié qu'en 1881, sur fond de nouvelles tensions franco-britanniques. Durant quarante ans, la mission s'imposa donc comme l'unique autorité de l'archipel, instaurant une véritable théocratie.

Tout en replaçant l'archipel dans le maelström des ambitions missionnaires et coloniales qui toucha l'ensemble de l'Océanie au $\mathrm{XIX}^{\mathrm{e}}$ siècle, Claire Laux (pp. 51-65) montre comment les missionnaires picpusiens tinrent l'archipel des Gambier à l'écart des 
« influences pernicieuses » (p. 51) des navigateurs et aventuriers européens en quête de richesses et de paradis terrestres ( $c f$. Christian Coiffier, pp. 25-33). La volonté du père Laval de préserver sa "cité idéale » des maux de l'Occident ( $c f$. Claire Laux, p. 53) en fit un opposant déclaré à la colonisation et ses relations avec les Européens approchant l'archipel furent souvent exécrables. À l'anticolonialisme du père Laval et des missionnaires picpusiens répondit bientôt un anticléricalisme colonial, largement porté par les représentants de la France qui présentèrent la colonisation comme une libération du poids missionnaire. La controverse faisait du lucre, de la dépravation et du déclin des populations autochtones ses principaux thèmes de débats. À partir des années 1870, la mission compta moins de missionnaires et fut affaiblie par les critiques diffusées par les détracteurs du Père Laval. Les îles Gambier illustrent bien les relations complexes qu'entretinrent, en Océanie, les missions avec le pouvoir colonial et qui souvent, ne se réduisirent pas à la collaboration du sabre et du goupillon.

Comme ailleurs en Océanie, l'action des missionnaires bouleversa profondément la société traditionnelle. Outre la conversion et la prohibition de la nudité, les missionnaires introduisirent de nouveaux éléments de la faune et de la flore (cochons, chèvres, bananiers, patate douce par exemple), définirent de nouveaux usages sociaux, des codes de lois et sanctionnèrent ceux qui ne s'y pliaient pas... Les missionnaires transformèrent également le paysage par l'édification de bâtiments religieux et l'organisation d'un habitat regroupé autour des lieux de culte. L'église NotreDame-de-la-Paix construite sur l'île d'Akamaru, en 1837, fut ainsi le premier édifice catholique bâti en Océanie; en 1839, la première église en pierre fut érigée sur l'île voisine d'Aukena. Les picpusiens mobilisèrent donc la population mangarévienne autour de la construction de dizaine de bâtiments dont la monumentalité et les décors travaillés participaient à l'enracinement de leur influence dans l'archipel tout en symbolisant la reconstruction de l'Église catholique après le Concordat de 1801. Les chrétiens mangaréviens furent également sollicités pour aider à la construction d'autres édifices, tel la cathédrale de Tahiti, en 18561857.

Un des grands intérêts de cet ouvrage collectif est de replacer Mangareva au cœur des grands enjeux européens dans le Pacifique tout en relevant les liens unissant, à partir de 1835 , l'histoire locale de la région aveyronnaise et de l'archipel des Gambier. L'origine lotoise du donateur du Rongo au musée de Cahors puis les missionnaires picpusiens sont au cœur de ces histoires croisées.

Entré dans la Marine en 1805, Joseph BonafousMurat, neveu du roi de Naples et beau-frère de Napoléon, fut nommé en 1832 capitaine de la frégate la Thisbée et commandant de la station du Brésil et des mers du Sud, en remplacement d'Abel DupetitThouars. Arrivée au Brésil en 1833, la Thisbée assuma plusieurs missions de reconnaissance et de protection des intérêts français sur les côtes sud-américaines avant d'appareiller pour la France, en mai 1834. Au terme de ce voyage et sans s'être rendu dans les îles
d'Océanie, Joseph Bonafous-Murat était en possession de onze objets polynésiens dont le Rongo de Mangareva qu'il versa au musée de Cahors, en janvier 1835. Si l'histoire de la collecte du Rongo et de sa transmission à Joseph Bonafous-Murat demeure mystérieuse, elle permit à la statuette aux yeux de lune d'échapper aux destructions des idoles qu'engagèrent les missionnaires nouvellement débarqués à Mangareva.

Les pères Laval et Caret furent rejoints, dès 1835 , par plusieurs religieux lotois et aveyronnais ; régions qui envoyèrent dans les années suivantes de nouveaux missionnaires aux Gambier et plus largement en Polynésie. Comment expliquer ces liens ? Après le Concordat et en pleine période de renouveau du catholicisme français, Cousin de Grainville, évêque de Cahors, Rodez et Montauban de 1802 à 1808, puis de Rodez de 1808 à 1822, permit l'implantation dans ces diocèses de communautés religieuses dont la congrégation des Sacrés-Cœurs de Picpus qui s'investit fortement dans l'instruction des jeunes garçons et filles. Or en 1833 le Pape confia à cette congrégation le vicariat apostolique de l'Océanie orientale. Il était naturel qu'une partie des vocations missionnaires suscitées par les picpusiens dans la région aveyronnaise, se tournassent vers ces îles. De même, il existe une correspondance entre l'ancrage forézien des pères maristes en charge de l'évangélisation de la Mélanésie, et l'origine forézienne d'une partie des missionnaires et des colons présents en Nouvelle-Calédonie. Entre 1834 et 1866, neufs missionnaires du Lot et de l'Aveyron partirent pour les Gambier, dont quatre périrent en mer en 1843. Par ailleurs, la congrégation était dirigée depuis 1849 par un Lotois, Monseigneur Bonamie, et le poste de Valparaiso fut occupé à deux reprises par un Lotois.

Si le choix de l'enchaînement des articles surprend parfois, cet ouvrage construit autour du parcours du dieu Rongo de Mangareva à Cahors, écrit l'histoire d'une rencontre entre Européens et Polynésiens, entre des missionnaires catholiques et la société mangarévienne. L'intérêt principal de l'ouvrage est de placer Mangareva, et plus largement l'archipel des Gambier, au cœur des enjeux missionnaires et coloniaux de l'Océanie du XIX ${ }^{\mathrm{e}}$ siècle tout en mettant en évidence ses liens avec l'histoire locale d'une région française. Certes, l'histoire du Rongo de Cahors, comme celle de la société mangarévienne d'avant le contact avec les Européens, garde de nombreuses zone d'ombre mais la redécouverte de la statuette a permis celle d'un épisode de l'histoire de l'archipel des Gambier dans un texte accessible au grand public.

\section{RÉFÉRENCES CITÉES}

Buck Peter Henry, 1938. Ethnology of Mangareva, Honolulu, Bernice Pauhai Bishop Museum, Bulletin 157.

LAVAL Honoré, 1968. Mémoires pour servir à l'histoire de Magareva, ère chrétienne, 1834-1871, Paris, Société des Océanistes, Publications de la Société des Océanistes 15.

Sarah MoHAmed-GaILlard, Paris, INALCO 